International Journal of Current Advanced Research

ISSN: O: 2319-6475, ISSN: P: 2319 - 6505, Impact Factor: SJIF: 5.995

Available Online at www.journalijcar.org

Volume 6; Issue 4; April 2017; Page No. 3349-3352

DOI: http://dx.doi.org/10.24327/ijcar.2017.3352.0269

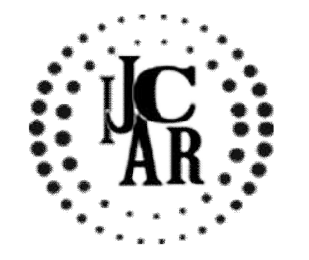

Research Article

\title{
APPROACH TO ACUTE ABDOMEN - A SURGEON'S PERSPECTIVE
}

\author{
Verma D.K., Tushar Aggarwal., Abhinav Verma and Pawan
}

Department of Surgery Indira Gandhi Medical College Himachal Pradesh India Shimla

\begin{tabular}{l}
\hline A R T I C L E I N F O \\
\hline Article History: \\
Received $8^{\text {th }}$ January, 2017 \\
Received in revised form $10^{\text {th }}$ February, 2017 \\
Accepted $22^{\text {nd }}$ March, 2017 \\
Published online $28^{\text {th }}$ April, 2017 \\
\hline
\end{tabular}

\section{Key words:}

Acute Abdomen, Surgeon's Perspective

\begin{abstract}
A B S T R A C T
The major constraints of surgeons in rural areas being either specialists of other fields (Anesthesia/Radiologist/Pathologist) being unavailable or no blood bank facilities in majority of rural hospitals. It is said, "Everybody's business is no body's business", but in rural setting, every business is surgeon's business. The key to best outcome in rural areas is, operate only when necessary and do the minimum possible, and at the same time, do not delay a necessary operation and do the maximum when indicated. The Aim of Surgery is that it should be safe, affordable, available near the point of demand and it should reduce mortality, morbidity and disability. It is thus of paramount importance to have adequate insight as to how to identify an acute condition and to provide adequate and timely help to tide it over. As said, "A stitch in time saves nine".
\end{abstract}

Copyright $@ 2017$ Verma D.K et al. This is an open access article distributed under the Creative Commons Attribution License, which permits unrestricted use, distribution, and reproduction in any medium, provided the original work is properly cited.

\section{INTRODUCTION}

The paradox of present times is that $70 \%$ of GDP in India is constituted by people who live in rural areas and urban slums of the country, who have little access to the basic modern surgical care, while $80 \%$ of hospital beds in India are in city hospitals, away from the demand of $70 \%$ of Indian population. The major constraints of surgeons in rural areas being either specialists of other fields (Anesthesia/ Radiologist/Pathologist) being unavailable or no blood bank facilities in majority of rural hospitals. It is said, "Everybody's business is no body's business", but in rural setting, every business is surgeon's business.

The key to best outcome in rural areas is, operate only when necessary and do the minimum possible, and at the same time, do not delay a necessary operation and do the maximum when indicated. The Aim of Surgery is that it should be safe, affordable, available near the point of demand and it should reduce mortality, morbidity and disability. A rural surgeon can be symbolized as an Infantry Officer, away from the limelight and glory that surrounds the Cardiac or Neurosurgeon, but someone has to agonize, sweat, bleed and wet his hands in human secreta \& secretions; all wars cannot be won by remote control with cruise missiles.

Approach to Acute Abdomen: In approaching a patient with acute abdomen, its wiser to follow old established principles rather than reinventing the wheel. Approach and management should be accurately tailored to the individual patient's

*Corresponding author: Verma D.K

Department of Surgery Indira Gandhi Medical College

Himachal Pradesh India Shimla disease- "Punishment should fit the crime; it is useless to fire indiscriminately in all directions". Excessive surgery, inappropriately performed and too late adds nails to the coffin. Zachary Cope (1881-1974) states, "Majority of severe abdominal pains which ensue in patients who have been previously fairly well and which last as long as six hours are caused by conditions of surgical importance". Or in other words, Acute abdomen refers to the abdominal pain of short duration that requires a decision regarding whether urgent intervention is necessary or not.

There is a Long list (20-30) of possible causes of acute abdominal pain- perforated peptic ulcer to port wine \& spider bites but multiple etiologies actually converge in to a small number of easily recognizable clinical patterns dictating a specific course of action.

\section{The various management strategies available are}

- Immediate Operation-"Surgery Now"

- Pre-operative Preparation \& Operation- "Surgery tomorrow morning."

- Conservative Treatment (Active Observation, I/V Fluids and Antibiotics)

- Discharge Home-(NSAP) Nonspecific abdominal pain-ill-defined, severe abdomen. Pain seeking medical attention, self-limiting-inv. Negative-Does not recur.

\section{Acute Abdomen can present as}

Abdominal Pain \& Shock: It is the most dramatic and least common. Common causes include, Ruptured abdominal aortic aneurysm, ruptured pregnancy, Intestinal Obstruction, Acute Pancreatitis \& Acute Mesenteric Ischemia. It requires 
immediate surgery without wasting any time in ancillary preparations and investigations.

Generalized Peritonitis: Patient presents with Diffuse Abdominal Pain, Sick \&Toxic look. Patient is motionless, with signs of peritonitis. Common Causes include Perforated ulcer, Perforated Appendix, Colonic perforation etc. Adequate management includes Pre-operative preparation \& OperationSurgery tonight. Exception is Acute Pancreatitis -Mild Epigastric Tenderness $+\mathrm{C} / \mathrm{F}$ of Ac. Peritonitis + Raised Serum Amylase, unnecessary Exploratory Laparotomy in Acute Pancreatitis may lead to disaster. "God put the

\section{Pancreas in the back because he did not want the Surgeons to mess with it."}

Localized Peritonitis: presents with symptoms and signs in one quadrant of Abdomen, common causes being, Acute Appendicitis, Acute Cholecystitis, Acute Diverticulitis. It is not an indication for Surgery tonight policy, exception is Acute Appendicitis. If the diagnosis is uncertain, it treated conservatively with I/V Fluids, Antibiotics.

Intestinal Obstruction: Patients present with Central colicky abdominal Pain, distension, constipation \& vomiting. Early \& more the Vomiting-More proximal is the site of obstruction while More marked the Distension-More distal the obstruction. Patients with previous history of surgery, presenting with adhesive obstruction can be managed conservatively. But we should proceed with surgery if there is history of fever, with leukocytosis and signs of Peritonitis. In cases of complete obstruction, with no gas in colon above peritoneal reflection, resolution chances are less, and surgery is the keystone to management.

Trauma: In Penetrating or blunt abdominal trauma, decision making is easier, it is not clouded by the altered sensorium due to head injury. Expensive, time consuming radiological investigations are unnecessary, except in stab injuries on back where CT is useful. Stab wounds are managed selectively, thereby avoiding negative laparotomies. Local Exploration is done, and in absence of peritoneal penetration, laparotomy is avoided. Laparotomy is done only if patient's condition deteriorates. In case of Gunshot wounds exploration is mandatory.

Gynecological: “Gynecologist's vision is usually limited by the boundaries of the bony pelvis." unless appendicitis is ruled out they will not diagnose anything as Gynecological. A surgeon catering to the rural population may find himself in a situation where due to lack of a gynecologist, he may have to treat patients otherwise falling under the purview of Gynecologists. History of Menstruation, Sexual activity and contraception is important as Pregnancy modifies the presentation of surgical conditions. General Surgeon should be in a position to handle acute abdominal pain in the reproductive years. Various causes of Acute abdomen related to obstetrics and gynecology posing a diagnostic dilemma for the surgeon includes, ectopic pregnancy, endometriosis, mittleschmerz disease, Pelvic inflammatory disease, twisted/ruptured ovarian cyst. Surgeon should be aware that in pregnant females, Nausea $\&$ Vomiting are common in $1^{\text {st }}$ Trimester, and also pregnant females may have Tachycardia, mild elevation of temperature and leukocytosis. In case of a Sick pregnant female- fetus is equally important but if mother is in distress -her benefits over ride the fetus. In First Trimester- Fetus is most susceptible to X-rays and drugs and abdominal operation may precipitate an abortion, and these are therefore best avoided. In Third trimester, operations can induce pre mature labor. Therefore, it is the Second trimester in which any Surgery, if required, is best tolerated.

Medical: Inferior wall Myocardial Infarction, Diabetic Ketoacidosis, Porphyria, Basal lobe Pneumonia may mimic Acute abdomen. But it is critical to differentiate these from various other surgical causes because Operating on these patients is an unfortunate medico legal occurrence and may prove to be a lethal mistake.

\section{Various Investigations required to diagnose a patient with acute abdomen includes}

- Abdominal X-ray

- Classical Surgeon's X-ray that show abnormal gas patterns or opacities

- Chest x-ray

- Free air is better seen than on Abdominal X-ray

- Tension Pneumothorax

- Lower lobe pneumonia

- Lung Metastasis/ Pleural Effusion

- Boerhaave's Syndrome

- Pneumothorax/ Pneumomediastinum and

- $\quad$ esophageal Rupture.

- USG

- Operator dependent

- Ac. Cholecystitis

- Pathology in female patients

- Acutely obstructed kidney as in ureteric stone.

- Acute Appendicitis

- Abdominal Fluid-Ascites, Pus or blood-localized or diffuse.

- FAST (Focused Abdominal Sonography for Trauma) IN Blunt Trauma Abdomen

- CT Scan

- Presumably not present in rural setting

- Not of much use (except in Acute Diverticulitis)

- Barium studies

- $\quad$ No use in Emergency

- Bacterium loves Barium- It protects them from the peritoneal macrophages leading to Peritonitis and IntraAbdominal abscesses.

- Once leaked, it is difficult to get rid of.

- Barium tends to stay and distort subsequent CT or Arteriography.

- Complete blood count.

- White Cell Count-Inflammatory Response-may be normal in Ac. Cholecystitis and Ac. Appendicitis.

- Low Hematocrit- Signifies Chronic or subacute Anemia.

- Routine blood biochemistry including serum amylase.

- LFT- Cholecystitis, Hepatitis or Cholangitis etc.

- KFT- Assess the level of Hydration.

\section{Optimization of a Patient with Acute Abdomen}

\section{When physiology is disrupted attempts at restoring Anatomy are futile}

The preparation of the patient for the surgery is as crucial as the surgery itself. 


\section{Double Edged Sword}

Wasting too much time trying to stabilize an exanguating patient is an exercise in Futility-Death

Rushing a hypovolemic patient suffering from intestinal obstruction is a recipe for disaster.

\section{Need for Optimization}

1. Volume depleted patients do not tolerate anesthesia and operation.

2. There is systemic vasodilatation depressing the compensatory anti shock physiologic mechanism.

3. Abdomen if opened without optimization-Intra peritoneal pressure declines-Pooling of blood in venous system-Decreased venous return-Dec. CO- Under resuscitated-Cardiac Arrest.

\section{Conditions requiring Optimization}

a. Sick looking patient-First impression

b. Tachycardia.

c. Tachypnea.

d. Hypotension.

e. Mental Confusion.

f. Poor peripheral perfusion.

g. High HB \& Hematocrit- Hemo-concentration and third space loss.

h. High specific gravity- $>1.039$ on urine analysis.

i. Electrolyte imbalance associated with pre renal azotemia (BUN 20:1)

j. Arterial Blood gas measurements- Give information about respiratory function.

k. Metabolic Acidosis-lactic acidosis-associated inadequate perfusion.

\section{Goals of Optimization}

\section{Correction of Hypovolemia}

1. Hypovolemia-Under perfusion of tissues-treated by volume expansion. In surgical patients, administer volume and more volume i.e. lot of fluids.

2. Not true in actively bleeding patients. Here optimization means immediate control of hemorrhageUntil this is achieved one should restrict the fluids and keep the patient moderately hypotensive

\section{Correction of sepsis}

Main goal is to improve the delivery of $\mathrm{O}_{2}$ to cells -There is direct relationship between cellular hypoxia and subsequent cellular dysfunction, SIRS, Organ failure and adverse outcome.

\section{Optimizing a patient}

1. Can be done anywhere with minimum facilities.

2. All we want is better oxygen delivery i.e. increased oxygenation of arterial blood and enhanced tissue perfusion.

Oxygenation- Hypoxia not only stops the motor, it wrecks the engine as well.

Restoration of Volume-Oxygen will arrive where it is needed only after blood volume is restored.

Crystalloids- Normal Saline or Ringer Lactate
Do not forget that rehydration with crystalloids may un mask chronic anemia as the hematocrit falls with volume expansion.

I/V Crystalloids@100-200ml/hr.

End point recognized by physical examination

Capillary refilling time-reliable clinical test

Urine output

UO 0.5 to $1 \mathrm{ml} / \mathrm{Kg} /$ Hour

We have to Aim for adequate UO with a CVP in the normal range up to $12 \mathrm{~cm}$ of water.

Message "As long as the UO is not adequate and CVP is lowPour in fluids".

Blood and blood products may need to be given- Indicated selectively to restore Oxygen carrying capacity in actively bleeding or chronic anemic patients and to correct clotting abnormalities if present. It includes

1. Whole blood.

2. Packed RBC.

3. FFP (Fresh Frozen Plasma)

4. Cryoprecipitate.

5. Platelet concentrate.

Best resuscitation in actively bleeding patients is surgical control of the source as pre-operative over resuscitation and transfusion increases the blood loss

\section{Two ends of spectrum}

- Uncontrolled hemorrhage requires immediate operation or Intervention after only partial fluid resuscitation or none at all.

- Intestinal Obstruction- requires complete resuscitation prior to operation.

- Most cases will fall somewhere in between which mean around 3 hours.

- Do not try to improve a non-responder beyond 6 hours -it is usually counter productive

"Never operate on a patient who is getting rapidly better or rapidly worse"

\section{Pre-Operative Antibiotics}

1. Therapeutic-if tissue infection is already established e.g. post-appendectomy

2. Prophylactic -in cases of Absence of Infection, with the objective of reducing anticipated evidence of infection which results from existing contamination during the operative procedure.

\section{CONCLUSION}

- Ac. Abdomen presents with few defined clinical patterns.

- $\quad$ Each Clinical pattern has set management options.

- Key to success is relevant investigations and optimization of the patient before any surgery.

- Unnecessary investigations are to be avoided.

- If in doubt, please refer the patient at the earliest to the higher center.

- "The operation is a silent confession to the surgeon's inadequacy"-John Hunter

- "The glory of surgeon is like that of actors, which lasts only for their own life time and can no longer be 
appreciated once they have passed away. Actors and surgeons are all heroes of the moment"

\section{References}

Schein's Common Sense Emergency Abdominal Surgery by Mosche Schein.

\section{How to cite this article:}

Verma D.K et al (2017) 'Approach To Acute Abdomen - A Surgeon's Perspective', International Journal of Current Advanced Research, 06(04), pp. 3349-3352.

DOI: http://dx.doi.org/10.24327/ijcar.2017.3352.0269 